\section{Alberto Enrique Rojas Calderón' 1 \\ Flor de María Pachas Barrionuevo $^{2}$}

${ }^{1}$ Cirujano-Dentista.

2Docente del Departamento Académico de

Odontología Social. Facultad de Estomatología.

Universidad Peruana Cayetano Heredia.

\section{Correspondencia}

Alberto Enrique Rojas Calderón

Coropuna 130 Urb. Maranga - Lima 32, Perú.

Teléfono: $452-4994$

e-mail: contactos2ae@yahoo.com

\title{
Perfil epidemiológico de salud oral e indicadores de riesgo en escolares adolescentes de la localidad de Cartavio (La Libertad, Perú)
}

Rojas-Calderón AE, Pachas-Barrionuevo FM. Perfil epidemiológico de salud oral e indicadores de riesgo en escolares adolescentes de la localidad de Cartavio (La Libertad, Perú). Rev Estomatol Herediana. 2010; 20(3):127-136.

\section{RESUMEN}

El presente estudio determinó la asociación del perfil epidemiológico de salud oral con indicadores de riesgo en escolares adolescentes de 10 a 15 años de edad, residentes en la localidad de Cartavio, La Libertad (2009). El diseño de estudio fue transversal, descriptivo. Se desarrolló un muestreo aleatorio estratificado proporcional (819 escolares). El método empleado para muestreo aleatorio estratificado proporcional (819 escolares). El método empleado para desarrolló según lo propuesto por la Organización Mundial de la Salud, para caries dental y enfermedad periodontal. Los indicadores de riesgo se obtuvieron mediante una encuesta estructurada. El análisis de variables incluyó pruebas chi cuadrado, U Mann Whitney, Kruskall Wallis, así como de regresión logística binaria como predictor de caries. La prevalencia y severidad de caries dental (CPO-D) en esta población fue de 70,1\% y 2,22 (DE=2,34), respectivamente. Las edades índice mostraron una prevalencia de 65,3\% (12 años) y $79 \%$ (15 años); el CPO-D fue de 1,94 (DE=2,27) y 3,39 (DE=3,07), a los 12 y 15 años respectivamente. El índice de significancia de caries (SIC) fue de 4,54 (12 años) y 6,96 (15 años). El valor CPITN con sextantes sangrantes fue más elevado a menor edad, contrario a la presencia de cálculos. El IHO-S fue 1,55 (DE=0,89). La edad del escolar, el grado de instrucción del jefe de familia (primaria), la visita y acompañamiento al dentista, así como el motivo restaurador, se comportaron como predictores de caries dental. El estudio epidemiológico identificó tendencias e indicadores de riesgo para su control y prevención.

Palabras clave: CARIES DENTAL / ENFERMEDAD PERIODONTAL / INDICADORES DE SALUD / ADOLESCENTE.

Epidemiological profile of oral health and risk indicators in adolescents of 10 to 15 years old from Cartavio (La Libertad), during 2009

ABSTRACT

The aim of the present study was to determine the association of oral health epidemiological profile with risk indicators in school adolescents of 10 to 15 years old, living in Cartavio (La Libertad), during 2009. This was a descriptive and cross sectional study. The total sample comprised of 819 school adolescents obtained by a random stratified proportional sampling. The method that was used to determine epidemiological profile was direct observation by recommendation of the World Health Organization. Risk indicators were obtained by using a structured survey. Data analysis was assessed by chi-square test, U Mann Whitney, Kruskall Wallis and binary logistic regression as a caries predictor. Prevalence and severity of dental caries (DMFT) in this population was $70.1 \%$ and $2.22(\mathrm{SD}=2.34)$ respectively. Index ages showed a prevalence of $65.3 \%$ and $79 \%$ at twelve and fifteen years old respectively. DMF-T was $1.94(\mathrm{SD}=2.27)$ and $3.39(\mathrm{SD}=3.07)$ at twelve and fifteen years old respectively. . Significant Caries Index (SIC) was 4.54 at twelve years old and 6.96 at fifteen years old. Community Periodontal Index (CPITN) with bleeding sextants was higher at younger ages. Contrary was the presence of calculus. OHI-S was $1.55(\mathrm{SD}=0.89)$. School adolescent age, Education status of family head (primary school), dental visits and treatment reason, were possible predictors to have dental caries. This epidemiological study is important to identify tendencies and risk indicators in some age groups to control and prevent disease.

Key words: DENTAL CARIES / PERIODONTAL DISEASE / HEALTH STATUS INDICATORS / ADOLESCENT.

complementan por largos períodos de tiempo (3). Los indicadores de riesgo están asociados a la ocurrencia de la enfermedad y se relacionan a los estudios transversales $(4,5)$. Su identificación permite tomar acciones de control y prevención dirigido a ciertos grupos $(1,6)$. En ese sentido, la adolescencia es una etapa de vida donde ocurren una serie de cambios por el proceso de crecimiento y desarrollo, la salud bucal no escapa a ello. La aparición de la caries dental y la enfermedad periodontal, de no ser controladas pueden causar un desequilibrio en los tejidos bucales, propiciando alteraciones funcionales (7). Diversas investigaciones reportan que factores de riesgo sociodemográficos, socioculturales, de estilo de vida y del sistema de salud oral, influyen en las prácticas de salud y repercuten sobre el bienestar aisladamente y muchos tienen cadenas complejas o eventos que se 
bucal, determinando la disponibilidad y prevención u orientación curativa $(3,8)$. En otros estudios, la aparición de caries dental está asociada al alto consumo de azúcares, mientras otros autores lo relacionan a indicadores de malnutrición (8-10).

La caries dental es una enfermedad infecciosa de naturaleza multifactorial, que se distribuye con distinta magnitud en diferentes comunidades $(1,11)$. La enfermedad periodontal es el resultado de la interacción huésped-parasito, en donde el tipo de placa junto a la respuesta inmune determina su severidad (12). Los índices epidemiológicos son indicadores que tratan de cuantificar los estados clínicos dentro de una escala graduada. El CPO-D y el CPITN son índices propuestos por la OMS para caries dental en dientes permanentes y enfermedad periodontal, respectivamente (13).

En las últimas décadas la salud bucal ha mostrado mejoría en países desarrollados; sin embargo, en América Latina, por carecer aún de muchos recursos, existe alta prevalencia de enfermedades bucales y el Perú no es una excepción. Este estudio buscó determinar la asociación del perfil epidemiológico de salud bucal con indicadores de riesgo en escolares de 10 a 15 años de edad, residentes en la localidad de Cartavio, La Libertad (2009).

\section{Material y métodos}

El presente estudio fue de tipo transversal y descriptivo. La población estuvo conformada por 1765 escolares de 10 a 15 años de edad matriculados en el año 2009, procedentes de instituciones públicas $(\mathrm{n}=3)$ y privadas $(\mathrm{n}=3)$ de Cartavio, localidad situada en el distrito de Santiago de Cao, provincia de
Ascope, departamento de la Libertad (Perú). Mediante un muestreo probabilístico estratificado proporcional se obtuvo una muestra de 819 escolares. Los criterios de selección fueron, pertenecer al grupo e instituciones educativas seleccionadas, residencia (>3 años), haber respondido a los cuestionarios de salud y poseer consentimiento o asentimiento informado, ausencia de tratamiento ortodóntico, ningún impedimento físico o mental y ausencia de alteración congénita. El $71,31 \%$ de escolares pertenecieron a instituciones públicas y 28,69\% a privadas.

El método para determinar las condiciones de salud bucal fue la observación directa y se emplearon los índices CPO y SIC para caries dental y CPITN en necesidad de tratamiento periodontal, según criterios de la OMS (6). La identificación de los indicadores de riesgo se obtuvo mediante una encuesta estructurada remitida a los padres. Las condiciones bucales se registraron en un formato ad hoc. Debido al tipo de estudio se requirió la carta de autorización del Comité Institucional de Ética de la UPCH. Un proceso de calibración interexaminador (InterE) e intraexaminador (IntraE) se desarrolló semanas previas a la recolección de datos. El examinador se consideró calibrado cuando obtuvo un índice Kappa mayor o igual a $80 \%$, siendo el CPO-D Inter $\mathrm{E}=0,930$ e IntraE $=0,934$; CPITN, InterE $=0,928$ e IntraE $=$ 0,930 ; IHO-S, InterE $=0,811$ e IntraE=0,853. La concordancia de Kappa se realizó mediante la aplicación del programa Excel 2003 (Microsoft Office) y el paquete estadístico SPSS 15.0.

La recolección de datos se efectuó en dos fases y se inició con la evaluación antropométrica (peso y talla), seguida de la obtención del índice de masa corporal (IMC) que empleó una tabla de percentiles para la edad CDC (Centers for Disease Control and Prevention); posteriormente se desarrolló la evaluación bucal (IHO-S, CPITN y CPO-D). Este examen se realizó con luz natural y equipo básico odontológico. Dos colaboradoras entrenadas prestaron apoyo para la organización de aulas y registro de datos.

\section{Resultados}

Se encontró una prevalencia de caries dental de $70,1 \%$ en la población evaluada que se incrementó con la edad. En relación al sexo no hubo diferencias estadísticamente significativas entre los 10 a 14 años; sin embargo se halló asociación a los 15 años de edad, siendo mayor la prevalencia en mujeres $(86,9 \%)$ que en varones $(70,7 \%)$. De esta manera, las mujeres tienen 2,8 veces más probabilidad que los varones de presentar este resultado $(p=0,030)$ $(\mathrm{OR}=2,75$ IC95\% [1,08-6,99]).

La severidad de caries dental (CPO-D) mostró una media total de 2,22 y tuvo tendencia a incrementarse con la edad, encontrándose diferencia estadísticamente significativa (Kruskal Wallis/p=0,000). No se halló asociación según el sexo.

El índice de significancia de caries (SIC) se incrementó con la edad (10 años SIC=3,14 / 15 años SIC=6,96); según el sexo, el promedio fue mayor en mujeres $(\mathrm{SIC}=5,06)$, que en varones (SIC=4,35) (Tabla 1).

El índice periodontal comunitario de necesidad de tratamiento (CPITN), según la edad, mostró una condición saludable estable, fluctuando entre $6,3 \%$ y $10,8 \%$. Se 
presentó una tendencia decreciente en la condición de sangrado, siendo a los 10 años 69,2\% y a los 15 años $21 \%$; fue ascendente respecto a la presencia de cálculos, entre 10 años (21,5\%) y 15 años (72,3\%) (Fig. 1). En relación al sexo, solo se mostró diferencias significativas en la condición periodontal a los 13 años de edad ( $p=0,004)$. La presencia de cálculos predominó tanto en mujeres
IEPRI, $\mathrm{p}=0$,267); sin embargo, hubo diferencias estadísticamente significativas al evaluar caries dental con la actividad laboral del jefe de familia en las IEPUB $(\mathrm{p}=0,003)$ (Tabla 2).

Indicadores socioculturales en relación a caries dental no mostraron diferencias estadísticamente significativas $(p=0,918)$ entre IEPUB e IEPRI, aunque la media fue mayor en IEPRI (CPO-D=2,26) que en IEPUB (CPO-D=2,20). Solo hubo asociación en el componente de piezas perdidas $(\mathrm{p}=0,003)$ y piezas obturadas ( $p=0,001)$. (Tabla 3).

Al evaluar el grado de instrucción del jefe de familia se encontró que la mayoría presentaba educación secundaria $\quad($ IEPUB $=81,8 \%$; IEPRI=53,6\%). En relación a caries dental (CPO-D), escolares con jefes

Tabla 1. Perfil epidemiológico de salud oral según edad y sexo, Cartavio-Perú, 2009.

\begin{tabular}{|c|c|c|c|c|c|c|c|c|c|c|c|c|c|c|}
\hline \multirow[t]{2}{*}{ Edad } & \multirow[t]{2}{*}{ Sexo } & \multicolumn{5}{|c|}{ Caries dental } & \multicolumn{8}{|c|}{ Necesidad de tratamiento periodontal } \\
\hline & & $\mathrm{P}$ & $\mathrm{P}^{*}$ & CPO-D & DE & $\mathrm{p}^{* *}$ & SIC & & $0(\%)$ & CPI1 & $(\%)$ & CPI2 & $(\%)$ & $\mathrm{p}^{*}$ \\
\hline \multirow[t]{3}{*}{10} & $\mathrm{~F}$ & 61,2 & 0,929 & 1,31 & 1,62 & 0,734 & 3,13 & 5 & $(1,2)$ & 36 & $(8,8)$ & 8 & $(2,0)$ & 0,487 \\
\hline & M & 62,1 & & 1,36 & 1,55 & & 3,16 & 5 & $(1,2)$ & 38 & $(9,3)$ & 15 & $(3,7)$ & \\
\hline & ST & 61,7 & & 1,34 & 1,58 & & 3,15 & 10 & $(1,2)$ & 74 & $(9,1)$ & 23 & $(2,9)$ & \\
\hline \multirow[t]{3}{*}{11} & $\mathrm{~F}$ & 59,4 & 0,466 & 1,59 & 1,82 & 0,168 & 3,67 & 8 & $(2,0)$ & 26 & $(6,3)$ & 30 & $(7,3)$ & 0,073 \\
\hline & $\mathrm{M}$ & 53,0 & & 1,12 & 1,99 & & 2,77 & 6 & $(1,5)$ & 40 & $(9,8)$ & 20 & $(4,9)$ & \\
\hline & ST & 56,2 & & 1,35 & 1,64 & & 3,22 & 14 & $(1,8)$ & 66 & $(8,1)$ & 50 & $(6,1)$ & \\
\hline \multirow[t]{3}{*}{12} & F & 65,9 & 0,878 & 2,07 & 2,49 & 0,729 & 4,86 & 9 & $(2,2)$ & 32 & $(7,8)$ & 44 & $(10,7)$ & 0,239 \\
\hline & M & 64,8 & & 1,81 & 2,03 & & 4,21 & 9 & $(2,2)$ & 23 & $(5,6)$ & 56 & $(13,7)$ & \\
\hline & ST & 65,3 & & 1,94 & 2,27 & & 4,54 & 18 & $(2,2)$ & 55 & $(6,7)$ & 100 & $(12,2)$ & \\
\hline \multirow[t]{3}{*}{13} & $\mathrm{~F}$ & 79,5 & 0,711 & 3,08 & 2,52 & 0,099 & 6,00 & 1 & $(0,2)$ & 27 & $(6,6)$ & 50 & $(12,2)$ & 0,004 \\
\hline & M & 76,9 & & 2,42 & 2,32 & & 5,14 & 8 & $(2,0)$ & 11 & $(2,7)$ & 46 & $(11,2)$ & \\
\hline & ST & 78,3 & & 2,78 & 2,45 & & 5,57 & 9 & $(1,1)$ & 38 & $(4,7)$ & 96 & $(11,7)$ & \\
\hline \multirow[t]{3}{*}{14} & $\mathrm{~F}$ & 79,5 & 0,873 & 2,70 & 2,23 & 0,274 & 5,25 & 3 & $(0,7)$ & 16 & $(3,9)$ & 54 & $(13,2)$ & 0,052 \\
\hline & M & 78,4 & & 2,24 & 1,85 & & 4,32 & 12 & $(2,9)$ & 15 & $(3,7)$ & 47 & $(11,5)$ & \\
\hline & ST & 78,9 & & 2,47 & 2,06 & & 4,79 & 15 & $(1,8)$ & 31 & $(3,8)$ & 101 & $(12,4)$ & \\
\hline \multirow[t]{3}{*}{15} & $\mathrm{~F}$ & 86,9 & 0,030 & 3,77 & 3,21 & 0,164 & 7,45 & 4 & $(1,0)$ & 15 & $(3,7)$ & 42 & $(10,2)$ & 0,615 \\
\hline & M & 70,7 & & 2,98 & 2,89 & & 6,47 & 4 & $(1,0)$ & 10 & $(2,4)$ & 44 & $(10,8)$ & \\
\hline & ST & 79,0 & & 3,39 & 3,07 & & 6,96 & 8 & $(1,0)$ & 25 & $(3,1)$ & 86 & $(10,5)$ & \\
\hline \multirow[t]{3}{*}{$10-15$} & F & 72,4 & & 2,46 & 2,52 & & 5,06 & 30 & $(7,3)$ & 152 & $(37,1)$ & 228 & $(55,6)$ & \\
\hline & M & 67,7 & & 1,98 & 2,13 & & 4,35 & 44 & $(10,8)$ & 137 & $(33,6)$ & 228 & $(55,6)$ & \\
\hline & $\mathrm{T}$ & 70,1 & $0,000 *$ & 2,22 & 2,34 & $0,000^{* *}$ & 4,71 & 74 & $(9,1)$ & 289 & $(35,4)$ & 456 & $(55,6)$ & \\
\hline
\end{tabular}

P:Prevalencia de caries dental. SIC:Índice de significancia de caries. F:femenino. M:masculino. ST:sub total. T:total. CPI:Índice periodontal comunitario, saludable (0), sangrante (1), cálculos (2). $\mathrm{p}^{*}$ :chi cuadrado. $\mathrm{p}^{* *}:$ U Mann Whitney. $\mathrm{p}^{* * *}$ :Kruskall Wallis.

(55,6\%) como en varones $(55,7 \%)$ (Tabla 1).

En relación a indicadores sociodemográficos, se evaluó la asociación de caries dental y el número de hermanos (mediana=2) según instituciones educativas públicas (IEPUB) y privadas (IEPRI); no se encontró diferencias estadísticamente significativas (IEPUB, $\mathrm{p}=0,181$ e IEPRI, $\mathrm{p}=0,145)$. En relación al total familiar (mediana=5), tampoco se encontró asociación (IEPUB, p=0,964;

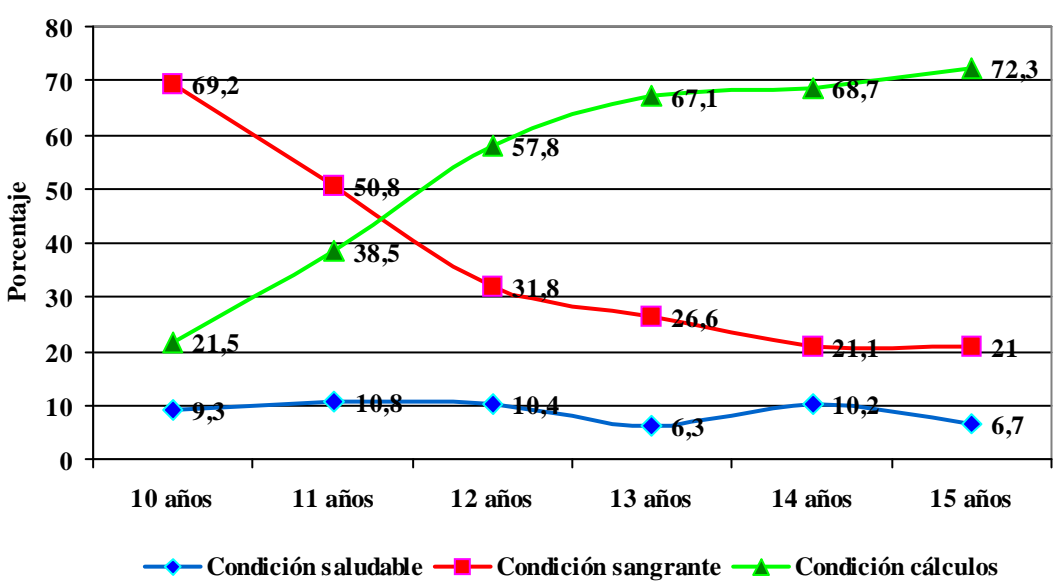

Fig. 1. Condición del índice periodontal comunitario de necesidad de tratamiento (CPITN), según edad, Cartavio-Perú, 2009. 
de familia con instrucción primaria presentaron los valores más altos en IEPUB $(2,80)$ e IEPRI $(3,83)$; con instrucción universitaria se presentó el valor mas bajo (IEPRI=1,71). Se encontró diferencias estadísticamente significativas al evaluar caries dental y el grado de instrucción del jefe de familia, tanto en IEPUB $(p=0,002)$ como en IEPRI $(p=0,048)$ (Tabla 3).

La valoración clínica de la condición de higiene oral en relación a la necesidad de tratamiento periodontal (CPITN) mostró que el 9\% de escolares presentó condición saludable, de ellos el 86,5\% presentó un índice de higiene oral simplificado (IHO-S) bueno; condición sangrante en $35,3 \%$, de ellos el $40,5 \%$ presentó IHO-S regular. Se halló presencia de cálculos en 55,7\%; de ellos, $70,8 \%$ mostró un IHO-S regular. Se encontró una relación muy significativa entre el IHO-S y la condición periodontal de necesidad de tratamiento $(p=0,000)$. El promedio general del IHO-S fue 1,55 ( $\mathrm{DS}=0,89$ ), la mayoría presentó una condición de higiene oral regular (54,9\%) y solo 5,4\%, mala (Tabla 4).

En cuanto a los factores de estilo de vida, conductas de salud bucal relacionadas a caries dental mostraron empleo masivo del cepillo y crema dental (96,6\% en ambos casos). La frecuencia de cepillado fue predominantemente dos veces al día (41,4\%). Hubo un consumo masivo de sal fluorada (100\%). En relación a la visita al dentista del escolar, el $58,1 \%$ tuvo su última atención dental el 2008 o años previos y el 22,2\% nunca asistió. La asistencia durante el año 2009 fue mayormente de una a dos veces (15,4\%) y predominó la atención preventiva (11,7\%). El 80,3\% nunca fue al dentista el 2009. Se encontró que el $64,7 \%$ de escolares refirió ser
Tabla 2. Indicadores sociodemográficos en relación a caries dental, en escolares de 10 a 15 años, según instituciones educativas de la localidad de Cartavio, La Libertad-Perú, 2009.

\begin{tabular}{|c|c|c|c|c|}
\hline CPO-D & Variables sociodemográficas & $\mathrm{n}$ & $(\%)$ & p \\
\hline \multirow[t]{2}{*}{ CPO-D } & IEPUB- Número de hermanos $\leq 2$ & 307 & $(52,7)$ & $0,181^{*}$ \\
\hline & Número de hermanos $>2$ & 275 & $(47,3)$ & \\
\hline \multirow[t]{2}{*}{ CPO-D } & Número de hermanos $\leq 2$ & 164 & $(69,2)$ & $0,145 *$ \\
\hline & Número de hermanos $>2$ & 73 & $(30,8)$ & \\
\hline \multirow[t]{2}{*}{ CPO-D } & IEPUB- Total familiar $\leq 5$ & 343 & $(58,9)$ & $0,964 *$ \\
\hline & Total familiar $>5$ & 239 & $(41,1)$ & \\
\hline \multirow[t]{2}{*}{ CPO-D } & IEPRI- Total familiar $\leq 5$ & 157 & $(66,3)$ & $0,267 *$ \\
\hline & Total familiar $>5$ & 80 & $(33,7)$ & \\
\hline CPO-D & IEPUB- Actividad laboral (jefe) & 582 & $(71,1)$ & $0,003 * *$ \\
\hline CPO-D & IEPRI- Actividad laboral (jefe) & 237 & $(28,9)$ & $0,225 * *$ \\
\hline
\end{tabular}

$\mathrm{p}^{*}: \mathrm{U}$ Mann Whitney. $\mathrm{p}^{* *}$ :Kruskall Wallis. IEPUB:Institución educativa pública. IEPRI:Institución educativa privada. Número de hermanos, mediana: 2 . Total familiar, mediana: 5.

Tabla 3. Indicadores socioculturales (instituciones educativas y grado de instrucción del jefe de familia) en relación a caries dental (CPO-D), CartavioPerú, 2009.

\begin{tabular}{lccccccc}
\hline & \multicolumn{1}{c}{ IEPUB } & \multicolumn{1}{c}{ IEPRI } \\
Indicador & $\mathrm{n} \quad(\%)$ & Media DE & $\mathrm{n} \quad(\%)$ & Media DE & $\mathrm{p}$ \\
\hline CPO-D & $582(71,1)$ & 2,20 & 2,32 & $237(28,9)$ & 2,26 & 2,39 & $0,918^{*}$ \\
$\mathrm{C}$ & & 1,93 & 2,14 & & 1,80 & 2,06 & $0,352^{*}$ \\
$\mathrm{P}$ & & 0,11 & 0,48 & & 1,19 & 0,50 & $0,003^{*}$ \\
$\mathrm{O}$ & & 0,16 & 0,65 & & 0,27 & 0,69 & $0,001^{*}$ \\
GI Primaria & $25(4,3)$ & 2,80 & 1,78 & $12(5,1)$ & 3,83 & 2,55 & \\
GI Secundaria & $476(81,8)$ & 2,28 & 2,36 & $127(53,6)$ & 2,28 & 2,28 & \\
GI Técnico & $63(10,8)$ & 1,49 & 2,19 & $64(27,0)$ & 2,22 & 2,52 & \\
GI Universitaria & $18(3,1)$ & 1,94 & 2,04 & $34(14,3)$ & 1,71 & 2,33 & \\
p & $0,002^{* *}$ & & & $0,048^{* *}$ & & & \\
\hline
\end{tabular}

IEPUB:Institución educativa pública. IEPRI:Institución educativa privada. DE:desviación estándar. GI:Grado de instrucción. p*:CPO-D, C, P, O. IEPUB-IEPRI (U Mann Whitney). p**:Grado de instrucción (GI). Institución educativa (Kruskal Wallis)

Tabla 4. Valoración clínica de la condición de higiene oral en relación a la necesidad de tratamiento periodontal (CPITN), Cartavio-Perú, 2009.

\begin{tabular}{lrccc}
\hline $\begin{array}{l}\text { IHO-S* } \\
\text { CPITN }\end{array}$ & $\begin{array}{l}\text { Bueno } \\
\mathrm{n}(\%)\end{array}$ & $\begin{array}{l}\text { Regular } \\
\mathrm{n}(\%)\end{array}$ & $\begin{array}{l}\text { Malo } \\
\mathrm{n}(\%)\end{array}$ & $\begin{array}{l}\text { Total } \\
\mathrm{n}(\%)\end{array}$ \\
\hline Saludable & $64(86,5)$ & $10(13,5)$ & $0(0)$ & $74(9)$ \\
Sangrado & $119(58,5)$ & $117(40,5)$ & $3(1)$ & $289(35,3)$ \\
Cálculos & $92(20,2)$ & $323(70,8)$ & $41(9)$ & $456(55,7)$ \\
Total & $325(39,7)$ & $450(54,9)$ & $44(5,4)$ & $819(100)$ \\
\hline
\end{tabular}

Chi-cuadrado de Pearson: 190,569 p=0,000. *IHO-S:1,55 ( $\pm 0,89)$. Índice de placa blanda:1,26 $( \pm 0,69)$. Índice de placa calcificada:0,30 $( \pm 0,35)$. acompañado por sus padres. El jefe de familia, en su mayoría, tuvo atención dental el 2008 o años previos (78,5\%) y solo el $6,7 \%$ nunca fue al dentista. La última visita al dentista, el motivo de la visita y el acompañante, mostraron asociación estadísticamente significativa en relación a caries dental $(\mathrm{p}<0,05)$ (Tabla 5).
Al evaluar el factor del patrón de consumo, no hubo diferencia estadísticamente significativa entre los indicadores del índice de masa corporal (IMC) con caries dental en relación a la edad, según prueba Kruskal Wallis ( $\mathrm{p}>0,05)$.

Respecto al factor sistema de salud oral, la mayoría de escolares $(53,4 \%)$ asistió a servicios 
particulares, 15,4\% a Essalud y solo el $9 \%$ al Minsa; se encontró que solo el 22,2\% nunca fue al dentista. Los escolares que nunca asistieron a un servicio dental mostraron un promedio CPO-D bajo $(1,58)$. Al evaluar las medianas de la caries dental con cada servicio de salud, se halló diferencias estadísticamente significativas (Kruskal Wallis, $\mathrm{p}=0,000$ ) (Tabla 6).

Un modelo predictivo se desarrolló a través de un análisis de regresión logística binaria, encontrándose asociación estadística significativa con la variable edad, mostrando una probabilidad de 1,3 veces más en presentar este desenlace ( $p=0,000$ [IC95\%: 1,1591,405]). Así mismo, al comparar el grado de instrucción del jefe de familia, se halló que los padres que tienen grado de instrucción primaria, tienen hijos con una probabilidad de 4,3 veces más de desarrollar caries dental. La visita al dentista mostró una asociación estadística significativa $(p=0,004$ [IC95\%: 1,180-2,415]) con una probabilidad de presentar la enfermedad en 1,7 veces. En relación al interés de los padres respecto a la atención dental, el acompañamiento de ellos indica la presencia de caries con una probabilidad de 2,3 veces ( $\mathrm{p}=0,021$ [IC95\%: 1,132-4,503]). De manera similar, el motivo de asistencia dental restaurador posibilita la presencia de caries, con una probabilidad de 6,3 veces $(\mathrm{p}=0,002$ [IC95\%: 1,94120,677]) (Tabla 7).

\section{Discusión}

La población del presente estudio correspondió a una muestra estratificada probabilística. El diseño transversal y descriptivo buscó determinar la asociación del perfil epidemiológico de salud oral con indicadores de riesgo, en escolares
Tabla 5. Conductas de salud bucal relacionadas a caries dental, en escolares y padres de familia, Cartavio-Perú, 2009. Conductas de salud bucal $\mathrm{n}$

\section{Del escolar}

Frecuencia de cepillado

No cepillado

Una vez

Dos veces

Tres o más (\%) $\mathrm{p}^{*}$

Uso de fluoruros

Consumo de sal fluorada

Última visita al dentista

Año 2009

2008 o años previos

Nunca fueron

Nunca en este año

Una o dos veces

Tres o mas

Motivo de visita al dentista 2009

Preventivo

Restaurador

Emergencias

Otros

Nunca fue al dentista

\section{De los padres}

Visita al dentista-acompañante

Padres

Otros

$530 \quad(64,7)$

$78 \quad(8,5)$

Solo

$29 \quad(3,5)$

Nunca fueron

$182(22,2)$

Visita al dentista-jefe de familia

Año 2009

$121 \quad(14,8)$

2008 o años previos

$643(78,5)$

Nunca fueron

$55 \quad(6,7)$

*p=Chi cuadrado

$\begin{array}{rrr}3 & (0,4) & >0,05 \\ 169 & (20,6) & \\ 339 & (41,4) \\ 308 & (37,6) \\ 819 & (100)\end{array}$

Tabla 6. Indicador del sistema de salud oral asociado a caries dental en relación a instituciones educativas, Cartavio-Perú, 2009.

\begin{tabular}{lrlrlrrr}
\hline & \multicolumn{2}{c}{ IEPUB } & \multicolumn{2}{c}{ IEPRI } & \multicolumn{2}{c}{ total } & CPO-D \\
Servicios de salud & $\mathrm{n}$ & $(\%)$ & $\mathrm{n}$ & $(\%)$ & \multicolumn{1}{c}{$\mathrm{n}$} & $(\%)$ & media \\
\hline Particular & 279 & $(47,9)$ & 158 & $(66,7)$ & 437 & $(53,4)$ & 2,51 \\
Essalud & 104 & $(17,9)$ & 22 & $(9,3)$ & 126 & $(15,4)$ & 1,94 \\
Minsa & 57 & $(9,8)$ & 17 & $(7,2)$ & 74 & $(9,0)$ & 2,51 \\
Nunca & 142 & $(24,4)$ & 40 & $(16,9)$ & 182 & $(22,2)$ & 1,58
\end{tabular}

$\overline{\text { IEPUB:Institución educativa pública. IEPRI:Institución educativa privada. Kruskal Wallis (p:0,000) }}$

Tabla 7. Regresión logística binaria en relación a caries dental e indicadores de riesgo. Cartavio, La Libertad-Perú, 2009.

\begin{tabular}{lrllllr}
\hline Indicadores de riesgo & Wald & gl & Sig & Exp (B) & \multicolumn{2}{c}{ IC95\% para EXP(B) } \\
& & & & & Inferior & Superior \\
\hline Edad & 24,575 & 1 & 0,000 & 1,276 & 1,159 & 1,405 \\
GIP & 6,915 & 1 & 0,009 & 4,335 & 1,453 & 12,937 \\
Visita al dentista & 8,204 & 1 & 0,004 & 1,688 & 1,180 & 2,415 \\
Acompaña (padres) & 5,349 & 1 & 0,021 & 2,258 & 1,132 & 4,503 \\
Motivo (restaurador) & 9,358 & 1 & 0,002 & 6,336 & 1,941 & 20,677 \\
\hline
\end{tabular}


de 10 a 15 años de edad, residentes en la localidad de Cartavio. La OMS define este grupo etario como adolescente y recomienda las edades de 12 y 15 años para establecer comparaciones, empleando criterios diagnósticos estandarizados $(5,6,13)$.

El estudio mostró una prevalencia de caries de $70,1 \%$ en la población evaluada, siendo 65,3\% a los 12 años y $79 \%$ a los 15 años. En la edad de 12 años, mayor fue lo reportado por Milciuviene et al. (Lituania, 2005) (14), Petersen et al. (Tailandia, 2001) (15) y Moura et al. (Brasil, 2008) (16). En relación a la edad de 15 años, Silva et al. (Brasil, 2007) (17), mostraron una prevalencia similar. La caries dental presentó una tendencia ascendente en relación a la edad, tal vez por el tiempo de exposición al ambiente oral, lo que es comparable a lo reportado por otras investigaciones (18-21). Respecto al sexo se encontró mayor prevalencia en mujeres, aunque sin diferencias estadísticamente significativas, similar a lo mostrado en otros estudios (19, 21, 22).

La severidad de caries dental general (CPO-D) fue 2,22; siendo 1,94 a $\operatorname{los} 12$ años de edad, comparable con el estudio de Moura et al. (16) y Jürgensen y Petersen (23). Este valor expresa el alcance de la meta propuesta por la OMS $(\mathrm{CPO}-\mathrm{D}<3)$, explicado seguramente por ser una población rural, además de otros factores de tipo protectivo. Silva reportó valores de 1,1; 1,2; 2,01 y 3,7 a los 10, 11, 12 y 15 años de edad respectivamente, debido probablemente a la presencia de fluoración en el agua de abastecimiento público (17); en el caso de la población de Cartavio se halló un consumo masivo de sal fluorada como parte de su estilo de vida, a la que se le reconoce, según la OMS, un efecto automático o pasivo de fluorización, independientemente de la intención de mejorar el estado de salud oral $(19,24,25)$. En el Perú, desde los años 80, el Ministerio de Salud garantiza la adecuada ingesta de flúor mediante el consumo de sal fortificada e inocua para la salud del consumidor (26).

El índice de significancia de caries (SIC) mostró la polarización de la severidad de caries dental. Cartavio registró valores de 4,44 y 6,77 a los 12 y 15 años de edad respectivamente. Son próximos los resultados mostrados por Silva et al. (17) y Herrera et al. (19) y a los 12 años de edad, siendo estos, poco más del $30 \%$ por encima de la meta propuesta por la OMS. Los resultados resaltan la limitación que ofrece la distribución del índice CPO-D y la conclusión engañosa que puede implicar.

El estado periodontal (CPITN) reveló que la condición de encías sangrantes fue mayor a menor edad (10 años=69,2\%); contrariamente hubo poca presencia de cálculos, que fue considerable a los 15 años (72,3\%). De manera similar, Marcantonio Junior y Santos (Brasil, 1998) (27) registraron que la condición de sangrado fue mayor a menor edad (10-11 años=49\%); así mismo, encontró gran presencia de cálculos a mayor edad (12-14 años=66,6\%). En la adolescencia, los cambios hormonales no causan gingivitis, sin embargo, influyen en la microcirculación, amplificando la respuesta inflamatoria en presencia de placa bacteriana (28). Albandar y Tinoco (29) refieren que la enfermedad periodontal presenta una asociación con la edad y de instalarse una gingivitis, puede ser el inicio de la pérdida de soporte en el periodonto. Por otro lado, Mello
(Brasil, 2006) (30) señaló que residir en áreas rurales, estudiar en escuelas públicas o ser varón, fueron factores asociados a sangrado gingival y cálculos, sin embargo, áreas rurales con sistemas productivos con una buena implementación agroindustrial están sujetos a mejores indicadores de salud bucal. Cartavio se ha desarrollado como uno de los complejos azucareros agroindustriales más grandes del Perú, favoreciendo a una mejor calidad de vida, que incide probablemente en su salud.

Indicadores sociodemográficos pueden contribuir al desarrollo de caries dental, es así que el número promedio de hijos es considerado un indicador de la carga familiar (8). En Cartavio se halló que $57,5 \%$ de escolares tenía uno a dos hermanos y un número total de cinco miembros por vivienda en poco más del $50 \%$, lo que se considera aceptable por el INEI (31) en su reporte 2007. No se observó asociación a caries dental probablemente por ese comportamiento demográfico. Aunque Delgado et al. (Perú, 2009) (32), encontraron una mayor probabilidad de presentar caries dental en adolescentes de 12 años de edad que residían en hogares pobres localizados en zonas marginales. En ese sentido, es conocido que la insatisfacción de necesidades básicas incluye hogares hacinados y se interpreta como una familia pobre, es así que algunos autores afirman que el incremento numérico de individuos viviendo en un hogar se relaciona a una alta experiencia de caries (16).

Las instituciones educativas evaluadas, públicas y privadas de la localidad de Cartavio, presentaron una prevalencia de caries homogénea. Tanto el promedio 
CPO-D como el componente de caries (C) no mostró diferencias significativas; contrario a los componentes perdidos (P) y obturados (O) en donde se encontró asociación, siendo mayor en colegios privados. Comparable fue el estudio de Traebert et al. (Brasil, 2001) (33), que encontró diferencias significativas entre escuelas públicas y privadas en el componente $\mathrm{O}$, con predominio en escuelas públicas, explicable por la eficiencia de los servicios dentales. Adecoya et al. (Nigeria, 2006) (34), mostraron diferencia significativa entre el tipo de escuela y la experiencia de caries dental, siendo menor en las públicas.

En relación a la condición de higiene oral, en Cartavio se obtuvo un promedio general regular (IHO$\mathrm{S}=1,55)$, siendo similar a lo encontrado por Rai (India, 2007) (35). Se ha comprobado que la deficiente higiene oral tiene una asociación muy alta con la gravedad de la inflamación gingival (27).

En cuanto al uso del cepillo, la gran mayoría (79\%) presentó una frecuencia de cepillado de dos o más veces, siendo masivo el uso de crema dental (99,6\%); aún así, se observó un promedio regular en el registro de placa blanda. Similarmente, Adekoya et al. (Nigeria, 2000) (34), también encuentra una alta frecuencia de cepillado $(76,6 \%)$ en escolares de 12 años de edad relacionado a una baja prevalencia de caries dental. Es probable que existan conductas de riesgo y estilos de vida no saludables que puedan ser modificados en los años de edad escolar. Los buenos hábitos adquiridos son sostenibles a edad temprana, es así que las prácticas de higiene oral pueden ser adoptadas e incorporadas fácilmente dentro de rutinas diarias, tanto en el hogar como en la escuela $(1,3,5,8)$.
Mas de las dos terceras partes de los escolares $(77,8 \%)$ visitó al dentista alguna vez, en donde solo el 19,7\% realizó su última visita durante el año 2009, mientras que el $58,1 \%$ lo hizo años previos. Se afirma que la visita al dentista, al menos una vez al año, reduce la probabilidad de desarrollar caries dental $(22,36)$. Aquellos que asistieron el año 2009, tuvieron en su mayoría consultas preventivas (chequeo dental, sellantes, fluorización) y solo un tercio de ellos recibió tratamiento restaurador. Poco más del $60 \%$ fue acompañado por sus padres, lo que significa el interés en la prevención de la salud bucal. Pachas (Perú, 2003) (8) encontró una respuesta similar en los niveles socioeconómicos bajo y medio alto; así mismo, Moura et al. (Brasil, 2008) (16) encontraron que $73,4 \%$ de escolares habían visitado al dentista por lo menos una vez, a diferencia del 26,6\% que nunca asistió; la mayoría fue el último año, lo que es favorable dentro del contexto de prevención.

En relación al patrón de consumo, los escolares de la localidad de Cartavio presentaron una prevalencia de $67,4 \%$ de peso adecuado y $10,4 \%$ tuvieron obesidad, sin embargo, no se encontró asociación con experiencia de caries en cada grupo etario; similar fue lo encontrado por Zelocuatecati et al. (Méjico, 2005) (37), en donde no hubo diferencias significativas entre la caries dental y el IMC en escolares de 11 a 15 años de edad. Contrariamente, Bailleul-Forestier et al. (Francia, 2007) (38), en un estudio transversal encontraron diferencias significativas entre caries dental y obesidad ( $p=0,002)$; así mismo, esta investigación refiere que en 1988, la prevalencia global en escolares de 10 a 17 años de edad procedentes de áreas rurales y urbanas en Francia fue de $15,3 \%$, siendo mayor en familias de bajos ingresos. El sobrepeso y la obesidad son considerados un problema de salud pública mundial $y$ se ha incrementado en la población adolescente e infantil $(9,37)$.

La población rural con seguro de salud (Minsa) en la provincia de Ascope asciende a 5438 habitantes y 2541 pueden acceder a Essalud (10), sin embargo en la población de estudio, poco más de la mitad frecuentan atención particular y de ellos, casi el $70 \%$ pertenecían a escuelas privadas. Probablemente la distancia no permite el acceso inmediato al servicio odontológico que brinda el Estado para recibir una atención adecuada, sin embargo existe cercanía y disposición de consultorios particulares. Solo la cuarta parte de escolares refiere haberse atendido en establecimientos de Essalud o Minsa. Se encontró que el 29,9\% de escolares no mostró experiencia de caries, lo que puede explicar el 22,2\% que nunca asistió a un servicio odontológico. A nivel poblacional, el resultado de la salud oral está relacionado a las características de los servicios de salud oral disponibles, pero también es considerada la conducta en las prácticas de higiene $(1,3,39)$.

El modelo predictivo de riesgo a desarrollar lesiones de caries en dientes permanentes permitió identificar los posibles indicadores que afectan a la población objetivo del estudio; sin embargo, cualquier modelo predictivo de una enfermedad tendría que ser lo más sensible y específico para identificar de manera sencilla, eficaz y clara a los individuos que están en riesgo de enfermar, con el objeto de dirigir las medidas preventivas correspondien- 
tes en el sentido adecuado (4). En el estudio, la edad tuvo 1,3 veces más riesgo de desarrollar la enfermedad, posiblemente por la mayor exposición al medio bucal de las piezas dentarias permanentes y por los cambios en la dieta debido al consumo de azúcares $(18,19,21)$. El ser acompañado al dentista por los padres genera una asociación significativa, con una probabilidad de 1,7 veces más de presentar caries, lo que muestra el interés de los padres de familia. Pachas (Perú, 2003) (8) encontró que dentro de las conductas de salud oral, el ser acompañado por los padres no solo favorece la prevención sino que demuestra su interés. De la misma forma, el tener padres con instrucción primaria genera más probabilidad de desarrollar este desenlace, comparado con los que presentan grados académicos más altos, como instrucción técnica o universitaria. Moura et al. (16) refieren que la educación permite acceder a una determinada ocupación laboral, posibilitando mayores ingresos; por esta razón, algunos la consideran como un indicador de la condición socioeconómica. La visita al dentista, el interés de los padres en acompañar a sus hijos, así como la atención dental con motivo restaurador fue un indicador de caries dental, probablemente con la intención de revertir este resultado y mejorar el estado de salud bucal.

Es necesario comprender que la modificación de estilos de vida y el acceso a los servicios de salud, desempeñan una función importante en la prevención de afecciones orales. El Perú presenta en muchas comunidades una alta prevalencia de caries dental y enfermedad periodontal, sin embargo, están sujetos a indicadores sociales distintos y a aspectos diferentes de salud pública. Cartavio no fue ajeno a estas diferencias $(1,5,10)$.

\section{Conclusiones}

La prevalencia de caries dental en la población de estudio de 10 a 15 años, así como su severidad, tuvo tendencia a incrementarse con la edad, sin asociación según el sexo, resultado similar mostró el SIC.

El CPITN, según la edad, mostró una tendencia decreciente en la condición de sangrado, siendo más elevada a los 10 años y menor a los 15 años; contrariamente, la presencia de cálculos aumentó con la edad. Respecto a los indicadores sociodemográficos, solo la actividad laboral del jefe de familia se asoció a caries dental. En relación a los indicadores de riesgo socioculturales se halló un promedio menor de la severidad de caries dental en IEPUB en relación a IEPRI. El grado de instrucción del jefe de familia que más prevaleció en ambas instituciones educativas fue la instrucción secundaria. Escolares con valores altos de caries dental fueron aquellos que tuvieron padres con instrucción primaria; mientras que los valores más bajos se observaron en escolares con padres de instrucción superior (técnica o universitaria).

El uso del cepillo y pasta dental así como el consumo de sal fluorada fue casi masivo. Se encontró una asociación significativa entre la condición clínica del CPITN y el IHO-S.

El sistema de salud oral mostró que la mayoría de escolares asistió a servicios particulares y solo la cuarta parte refirió atenderse en Minsa o Essalud. Menos de la cuarta parte de escolares nunca fue al dentista. El modelo predictivo de riesgo a desarrollar lesiones de caries en dientes permanentes sanos identificó como predictores de caries dental a la edad, hijos de padres con grado de instrucción primaria, la vista al dentista, el acompañamiento del padre a la visita dental y el motivo restaurador.

\section{Agradecimientos}

Los autores expresan su reconocimiento a la licenciada Leila Castillo V. (Trabajo Social-Pontificia Universidad Católica del Perú) por su participación en la coordinación con las instituciones educativas de la localidad (Cartavio, La Libertad), así como a las autoridades respectivas. Mención especial al Mg Marco Alarcón P. por su colaboración en el proceso de calibración (CPITN) y al Mg Luis Arriola G. como co-asesor.

\section{Referencias bibliográficas}

1. Daly B, Watt R, Batchelor P, Treasure E. Essential dental public health. New York: Oxford University Press; 2003.

2. Dever A. Epidemiología y administración de servicios de salud. Ed OPS/OMS. Maryland: Aspen Publisher Inc; 1991.

3. Petersen PE. Sociobehavioural risk factors in dental caries international perspectives. Community Dent Oral Epidemiol. 2005; 33(4):274-9.

4. Mattos M, Melgar M. Riesgo de caries dental. Rev Estomatol Herediana. 2004; 14(1-2):101-6.

5. David J. Dental caries among adolescents-Implications for planning oral health services in India and Norway. (PhD thesis). Bergen: University of Bergen; 2006.

6. World Health Organization. Oral Health Surveys-Basic Methods. 4th ed. Geneve: World Health Organization; 1997. 
7. Greene JC, Suomi JD. Epidemiology and public health aspects of caries and periodontal disease. J Dent Res. 1977; 56 Spec No:C20-6.

8. Pachas F. Factores sociodemográficos, conductas en salud oral de escolares y padres, uso de servicios dentales asociados a experiencia de caries dental en dentición permanente de escolares de dos niveles socioeconómicos de Lima Metropolitana. (Tesis Mg. en Estomatología). Lima: Universidad Peruana Cayetano Heredia; 2003.

9. Mota-Sanhua V, OrtegaMaldonado M, López-Vivanco JC. Factores familiares asociados con el estado de nutrición y la salud oral en adolescentes Rev Med Inst Mex Seguro Soc. 2008; 46(3):253-60.

10. Henostroza G. Diagnóstico de caries dental. Lima: Universidad Peruana Cayetano Heredia; 2005.

11. Pérez A, Quenta E, Cabrera A, Cárdenas D, Lazo R, Lagravere M. Caries dental en dientes deciduos y permanentes jóvenes. Diagnóstico y tratamiento conservador. Lima: Universidad Peruana Cayetano Heredia; 2004.

12. Kinane DF, Podmore M, Murray MC, Hodge PJ, Ebersole J. Etiopathogenesis of periodontitis in children and adolescents. Periodontol 2000. 2001; 26:54-91.

13. WHO Oral Health Country/Area Profile Programme (CAPP). Malmö, Sweden; 2009. Disponible en: http:// www.whocollab.od.mah.se/ index.html - CAPP.

14. Milciuviene S, Bendoraitiene E, Andruskeviciene V, Narbutaite J, Sakalauskiene J, Vasiliauskiene I,
Slabsinskiene E. Dental caries prevalence among 12-15-yearolds in Lithuania between 1983 and 2005. Medicina (Kaunas). 2009; 45(1):68-76.

15.Petersen PE, Hoerup N, Poomviset N, Prommajan J, Watanapa A. Oral health status and oral health behaviour of urban and rural schoolchildren in Southern Thailand. Int Dent J. 2001; 51(2):95-102.

16. Moura C, Cavalcanti AL, Medeiros Bezerra PK. Prevalência de cárie dentária em escolares de 12 anos de idade, Campina Grande, Paraíba, Brasil: enfoque socioeconômico. Rev Odonto Ciênc. 2008; 23(3):256-62.

17. Silva SRC, Fernandes CE, Alves RX. Condição da saúde bucal de escolares e pré-escolares, Araraquara - SP, 2004. Rev Odontol UNESP. 2007; 36(2):145-50.

18. Santos Martí J, Rubio García B, Santos Bistué C, Rubio Calvo E. Índices epidemiológicos de caries de la población escolar atendida en el Centro de Salud de Barbastro. Av Odontoestomatol. 2005; 21(1):355-60.

19. Herrera MS, Medina-Solis CE, Maupomé G. Prevalencia de caries dental en escolares de 612 años de edad de León, Nicaragua. Gac Sanit. 2005;19(4):302-6

20. Yabao RN, Duante CA, Velandria FV, Lucas M, Kassu A, Nakamori M, Yamamoto S. Prevalence of dental caries and sugar consumption among 6-12$\mathrm{y}$-old schoolchildren in La Trinidad, Benguet, Philippines. Eur J Clin Nutr. 2005; 59(12):1429-38.

21. Medina C, Cerrato J, Herrera M. Perfil epidemiológico de la caries dental y enfermedad periodontal, en Nicaragua, año 2005. Ed Universitaria Universitas 2007; 1:39-46.

22. Almerich-Silla JM, MontielCompany, JM. Encuesta de salud oral en la población infantil de la Comunidad Valenciana, España (2004). Med Oral Patol Oral Cir Bucal. 2006; 11(4):369-81.

23. Jürgensen N, Petersen PE. Oral health and the impact of sociobehavioural factors in a cross sectional survey of 12-year old school children in Laos. BMC Oral Health. 2009; 9:29.

24. Velázquez $\mathrm{O}$, Vera $H$, Irigoyen ME, Mejía A, Sánchez TL. Cambios en la prevalencia de la caries dental en escolares de tres regiones de México: encuestas de 1987-1988 y de 1997-1998. Rev Panam Salud Publica. 2003; 13(5):320-6.

25. Jones S, Burt BA, Petersen PE, Lennon MA. The effective use of fluorides in public health. Bull World Health Organ. 2005; 83(9):670-6.

26. Ministerio de Salud, Resolución Ministerial $\mathrm{N}^{\circ}$ 961-2006. Reglamento técnico para la fortificación de la sal para consumo humano con yodo y flúor. Diario El Peruano, 16 de Octubre del 2006.

27. Marcantonio Júnior E, Santos FA. Avaliação das condições e necessidades de tratamento periodontal em escolares da zona rural da região noroeste do estado de São Paulo - Brasil. Rev Odontol UNESP. 1998; 27(2):449-58.

28. Murrieta-Pruneda JF, JuárezLópez LA, Linares-Vieyra C, Zurita-Murillo V, MeléndezOcampo AF, Ávila-Martínez CR, Nava-Delgado CM. Prevalencia de gingivitis asociada a la higiene 
oral, ingreso familiar y tiempo transcurrido desde la última consulta dental, en un grupo de adolescentes de Iztapalapa, Ciudad de México. Bol Med Hosp Infant Mex. 2008; 65(5):367-75.

29. Albandar JM, Tinoco EM. Global epidemiology of periodontal diseases in children and young persons. Periodontol 2000. 2002; 29:153-76.

30. Mello T. Diferenciais de saúde em áreas urbanas e rurais: prevalência de cárie dentária e condições gengivais no Estado de São Paulo. (PhD Thesis). Brazil: Faculdade de saúde pública da USP; 2006.

31. INEI. Censos nacionales 2007: XI de población y VI de vivienda. Perfil sociodemográfico del Perú. 2da. Ed. Lima: Centro de Edición de la Oficina Técnica de Difusión del INEI; 2007.

32. Delgado-Angulo EK, Hobdell
MH, Bernabé E. Poverty, social exclusion and dental caries of 12year-old children: a crosssectional study in Lima, Peru. BMC Oral Health. 2009; 9:16.

33. Traebert JL, Peres MA, Galesso ER, Zabot NE, Marcenes WS. Prevalência e severidade da cárie dentária em escolares de seis e doze anos de idade. Rev Saúde Pública. 2001; 35(3):2838.

34. Adekoya-Sofowora CA, Nasir WO, Oginni AO, Taiwo M. Dental caries in 12-year-old suburban Nigerian school children. Afr Health Sci. 2006; 6(3):145-50.

35. Rai B, Jain R, Duhan J, Anand S. Relationship between dental caries and oral hygiene status of 8 to 12 year old school children. Internet J Epidemiol. 2007; 4(1).

36. Tramini P, Molinari N, Tentscher M, Demattei C, Schulte AG. Association between caries experience and body mass index in 12-year-old French children. Caries Res. 2009; 43(6):468-73.

37.Zelocuatecatl AA, Ortega MM, Fuente HJ. Asociación entre el índice de masa corporal y las condiciones bucales en escolares. Rev Odont Mex. 2005; 9(4):185-90.

38. Bailleul-Forestier I, Lopes K, Souames M, Azoguy-Levy S, Frelut ML, Boy-Lefevre ML. Caries experience in a severely obese adolescent population. Int J Paediatr Dent. 2007; 17(5):35863.

39. Nieto Garcia VM, Nieto Garcia MA, Lacalle Remigio JR, AbdelKader Martin L. Salud oral de los escolares de Ceuta: influencias de la edad, el género, la etnia y el nivel socioeconómico. Rev Esp Salud Publica. 2001; 75(6):541-50. 